\title{
LA COMPETITIVIDAD DE LOS PARTIDOS POLÍTICOS EN BAJA CALIFORNIA EN LAS ELECCIONES DE 1997
}

\author{
Por \\ Leopoldo Martínez Herrera*
}

\begin{abstract}
RESUMEN
En México, la transición democrática, como estrategia del cambio político, ha cobrado una alta dinamicidad; además, los partidos han tenido que someterse a la evaluación de los ciudadanos, esto último derivado de la recuperación de la autonomía de la conciencia de la sociedad. Bajo los anteriores supuestos, la competitividad político-electoral, a la vez que es un método para medir la capacidad de ofensiva y de disputa de los partidos políticos en las actuales circunstancias, es también una condición para su actuación en medio de un sistema electoral moderno, democrático, y por ello, equitativo. Un caso ilustrativo lo constituye Baja California, que el 6 de julio de 1997, eligió a seis representantes distritales a la Cámara de Diputados de la Nación. El resultado de dicho evento se presenta en este artículo a la luz de la medición de la competitividad de los partidos que contendieron en ella a través de cinco indicadores: a) competitividad simple, b) margen de victoria del PAN respecto al PRL, c) potencia opositora, d) número de partidos y e) expansión del sistema electoral.
\end{abstract}

\begin{abstract}
In Mexico, the democratic transition, as a strategy for the political change, has gained a high effectiveness, also the parties have had to submit themselves to the evaluation of the citizens due to the recuperation of the liberty of society's conscience. Under the above circumstances, the electoral-political competition, once it is a method to measure the capability of effectiveness and of dispute of the political parties in the actual circumstances, it is also a condition for their act within a modern, democratic and equal electoral system. An illustrative case is Baja California, that on July 6, 1997, elected six representatives for the House of Representatives of the Nation. The result of this event is presented in this article openly by the measure of the competition of the parties involved by the following indicators: a) simple competition, b)margin of victory between PAN in relation to PRL, c) the power of the opposing party, d) number of political parties, and e) expansion of the electoral system.
\end{abstract}

* Vocal ejecutivo de la Junta Distrital del 01 Distrito Federal Electoral. 


\section{INTRODUCCIÓN}

¿Por qué interesa estudiar el grado de competencia o la competitividad de un sistema de partidos? Pensamos que este trabajo debe iniciar su reflexion a partir precisamente de esta pregunta.

A raíz del inicio de la presente década, algunos escritores han desarrollado elaboraciones teóricas, respecto a las posibilidades de que existan ya en el horizonte de nuestro sistema electoral, condiciones favorables para que en este país se realicen lo que se puede llamar elecciones completamente competitivas, o que al menos tendencialmente se avizoren algunos rasgos que denoten tal condición.

El estudio de algunas de las características del sistema político mexicano durante la última década, nos habla de que un esfuerzo del régimen para gestar condiciones mínimas de competencia, ha corrido al paralelo de los avances que se han realizado a partir de 1990, en el ámbito de las reformas electorales que hasta la fecha han operado.

La fase intensa de la reforma electoral que comprende el periodo 1993-1996, nos ofrece, con toda claridad, que el ámbito de las condiciones de la competencia electoral constituyó uno de los nudos problemáticos hacia el cual se remitió el debate, para llegar, paulatinamente y en un proceso de decantamiento, a circunstancias en las cuales se puede asumir que son relativamente las más favorables que se han logrado en la historia electoral del país.

Sin embargo, llegar a esta situación ba implicado una dura batalla que han sostenido las diversas fuerzas políticas del país, así como los diferentes agrupamientos civiles de intelectuales y escritores políticos. El escenario deseado fue siempre la aspiración de gestar condiciones favorables de la competencia en las cuales, ninguna organización política, en plena contienda, pudiera utilizar factores y medios que lo pusieran en una ventaja insuperable con respecto al resto de los partidos políticos.

Para los efectos anteriores, parecía que la evaluación histórica de nuestro sistema abonaba los cambios, pues México, desde bacía tres lustros, había avanzado de ser un país con un sistema de partido hegemónico, hacia otro de partido predominante, e incluso que se dirigía a paso veloz, hacia uno de pluralismo moderado, ante la presencia de tres fuerzas (PRl, PAN y PRD) ${ }^{1}$ ya influyentes durante el proceso electoral de 1994.

\footnotetext{
1 Partido Revolucionario Institucional, Partido Acción Nacional y Partido de la Revolución Democrática, respectivamente.
} 
No obstante lo anterior, resulta necesario precisar que esta expresión del fenómeno del pluralismo moderado, aún no constituye una forma madura ni consistente, pues como observaremos más adelante, se manifiesta claramente en determinadas zonas del pais; en cambio, en otras, resulta evidente una especie de bipartidismo consistente.

Ante esta manifestación, sólo podemos opinar en el sentido de que es posible se esté fraguando una tendencia general bacia el pluralismo moderado, cohabitando con zonas territoriales en las cuales aún persiste un sistema de partido predominante, con un bipartidismo todavía consistente.

Esta fisonomía constituye una de las características propias de países en procesos transicionales, formas duales de convivencia de modelos y pautas de conductas económicas, políticas y sociales. De tal manera que, como señala Sartori (1987), medir las condiciones de la competencia, nos dirige a evaluar también otros aspectos sustanciales del sistema político, a saber: a) el grado de fragmentación en que se encuentra el sistema de partidos en el país, y b) el grado de distribución del poder entre las diversas fuerzas políticas de un país.

Para el caso que nos ocupa, nos interesa por un lado, analizar ex post, el grado de competitividad que han manifiestado los tres partidos políticos ya mencionados con anterioridad (PRI, PAN y PRD), durante el proceso electoral de 1997, en el cual se renovó la integración de la Cámara de Diputados, y parcialmente la Cámara de Senadores. En este análisis nos preocupa, sobre todo, precisar la relación de competitividad del PRI y del PAN, por ser éstos los partidos que tradicionalmente han disputado el poder en Baja Califomia; fenómeno que se repite con bastante claridad para las elecciones del 28 de junio de 1998.

Como necesidad metodológica, e intención de cristalizar el esfuerzo de medir el grado de competitividad del sistema de partidos en Baja Califomia, de entrada tuvimos que eliminar del análisis a los partidos PC, PVEM, PDM, PPS, $\mathrm{PT}^{2}$ y parcialmente el PRD; este último, que no obstante haber tenido altos grados de competitividad en el Distrito Federal y en algunas entidades de la república, en nuestro estado su avance electoral no logra, en esta última elección, cambiar sustancialmente la correlación de fuerzas políticas, incluso, ni la correlación de fuerza electoral, como veremos con la aplicación de Ios índices de competitividad que hemos utilizado: diriamos, retomando a Sartori, que si acaso, el PRD, en esta última olección, sólo demostró poseer una de las dos variables como partido

2 Partido Comunista, Partido Verde Ecologista de México, Partido Demócrata Mexicano, Partido Popular Socialista y Partido del Trabajo, respectivamente. 
significante; es decir, únicamente tiene una capacidad relativa para modificar la relación de competencia de los dos partidos opositores relevantes: PRI y PAN (Sartori lo llama capacidad de chantaje).

Los indicadores de competitividad que utilizamos son: a) triunfos obtenidos, b) margen de victoria, c) potencia opositora, d) número de partidos y, e) expansión del sistema electoral. Estos indicadores permitieron determinar la capacidad de competencia con la que potencialmente llegaron al proceso electoral, y el resultado que obtuvieron de acuerdo con la capacidad aplicada, ya que, de nueva cuenta, retomando al autor italiano, finalmente la única medición de competitividad que podemos aplicar a los partidos políticos, es sólo por sus resultados (en este caso, los únicos resultados que cuentan son los votos y los escaños obtenidos).

\section{DEFINIENDO LA COMPETITIVIDAD}

La competencia, por ejemplo, una estructura competitiva de mercado, es importante como medio de proteger y beneficiar al consumidor, tanto al consumidor económico como al político. (Sartori, 1987:262)

La sentencia de Sartori resulta de un gran valor prescriptivo, por cuanto hace a una sociedad de mercado pleno, en el cual la gran parte del cosmos societario se ha mercantilizado. Esto sucede obviamente en las sociedades de capitalismo desarrollado; ello, sin embargo, no excluye a los países que, retomando el modelo liberal-democrático, se encuentran en una etapa de transición que los llevará de una sociedad semiautoritaria hacia otra democrática, como es nuestro caso.

La aseveración de Sartori, por otra parte, se inscribe en la égida de la ingenuidad evidente, pues parecería que ambos mercados -el de la economía y el de la política - sólo tienen como línea rectora, el principio samaritano de la búsqueda y protección de los demás. Lo anterior es cierto, sólo si y a condición de que, otra parte importante de ambos mercados, los que ostentan la fracción dominante de éste, obtengan también un alto beneficio y utilidad política que les permita conservar y proteger su posición privilegiada, y reproducir el sistema que evidentemente les beneficia.

Esto resulta más cierto en donde existen niveles de desigualdad verdaderamente notorios - incluso de actores políticos - y cuya inequidad de origen otorga todavía desventajas reales a algunos de ellos, gracias a un proceso acumulativo histórico. Ésta es la actual situación de nuestro país $\mathrm{y}$, consecuentemente, con mayor o menor vigencia, en algunas localidades y regiones. 
Lo que sí resulta necesario plantear como una premisa plausible, es que el debate respecto a la competencia de la actividad político-electoral, ya comienza a ser un tema recurrente en nuestro país, lo cual es sintomático de un sistema de partidos que se desplaza en el itinerario de los cambios, de un sistema de partido único, pasando por el modelo hegemónico y tocando los límites de partido predominante, para ubicarse en algunas regiones y localidades en el umbral de un sistema de pluralismo moderado, con la presencia importantemente competitiva del PAN, PRI Y PRD.

Es este tránsito histórico por los distintos modelos de partidos, lo que convierte al análisis de la capacidad competitiva de los institutos políticos, en un asunto ya de relevante interés, no solamente para los círculos académicos o agentes especializados en tal asunto, sino también para aquéllos que habrán de abrevare interesarse, sin duda, en su capacidad real para competir, así como en la evaluación objetiva que pueda realizarse de su desempeño político-electoral, gracias a sus campañas.

Una de las preocupaciones en los países inmersos en procesos transicionales es, precisamente, la medición de un fenómeno doble: el grado de fragmentación del sistema de partidos políticos, así como el grado en que se está manifestando la distribución del poder político. Lo anterior obedece a la idea de que, justamente, los sistemas electorales son democráticos en la medida en que las opciones políticas organizadas se pluralizan hasta un cierto grado, el cual no debe llegar a los límites de ta fragmentación atomizada.

Por otra parte, la competencia real se manifiesta cuando la disputa se desarrolla entre partidos con presencia, de cuyo resultado se deriva una determinada asignación de poder a cada uno de ellos; es decir, la competencia de un sistema se demuestra "en el sentido de acercarse a una distribución casi igual de fuerza entre los partidos principales". (Sartori, 1987:260)

Con todo y una vez expuesto el motivo por el cual resulta pertinente adentrarnos en las manifestaciones del fenómeno de la competitividad, dado que ésta nos muestra la expresión de otros fenómenos que ya hemos señalado, conviene precisar los términos que ayudan a clarificar el concepto de interés.

Para algunos autores políticos, parece que no hay una diferencia - ni tiene sentido buscarla- entre el concepto de competitividad y el de competencia. Ésta parece ser la posición de Rouquie cuando señala: " $¿$ Hay que decir que todas las elecciones son más o menos no competitivas? Por cierto, que no; o más bien sí, si entre el más y el menos se entiende que hay una diferencia de naturaleza". (Rouquie, 1986:157) 
Bajo este punto de vista, existe una noción normativa negativa, la cual parece sentenciar que todos los sistemas electorales son originariamente no competitivos; por ello, no existe un margen permisible entre el concepto y la práctica. Es decir, la diferencia natural de los actores políticos determina la falta de competitividad, tanto de una posible teoría y de reglas de juego de la competencia, como de su práctica. En esta perspectiva no existe diferencia entre competencia y competitividad, entre reglas de juego $y$ el juego mismo.

Lo anterior viene a colación porque esta diferencia sí forma parte de las preocupaciones del presente análisis, como lo describiremos a continuación.

Para nosotros, a diferencia de otros países, resulta importante darle seguimiento analítico a este problema de las reglas de juego que devienen normas legales, pues constituyen las llamadas condiciones de competencia.

Una de las señales de avance de un país en su tránsito hacia la democracia la constituyen, sin duda, las condiciones de la competencia, pues implica dos cosas: por un lado, el reconocimiento de ciertas reglas que flexibilizan la contienda política entre sus actores, lo cual significa, sin duda, la cesión de un campo de lucha política por parte del régimen; y por el otro, denota la inminente presencia (aunque no infalible) de otros actores políticos, sin los cuales no se propiciarían dichas condiciones de la competencia. Tal es el caso de nuestro país, por ello el interés en establecer una diferencia clara entre la competencia y la competitividad.

Precisamente es Sartori quien rescata la necesidad de tal diferencia, en el marco de caracterizar los distintos sistemas de partidos, ya que con este fin, una de las metas es la determinación del número real de partidos que tienen una importancia significativa en los distintos modelos.

De becho, estamos empleando dos conceptos: i) competencia, y i) competitividad. La competencia es una estructura o una regla de juego. La competitividad es un estado concreto del juego. (Sartori, 1987:260)

Las ideas del autor italiano nos dan claridad sobre la diferencia de los dos conceptos: diríamos, siguiendo el apunte anterior, que la competencia, así como sustantivo, es un conjunto de atributos que, bajo una concepción moral de equidad e igualdad, posee el sistema electoral cuyos beneficios conceptuales y de principios favorecen potencialmente al conjunto de actores políticos; lo cual no quiere decir que las reglas de juego establecidas (competencia) sean determinantes en una contienda político-electoral. 
La estructura de la competencia garantiza una serie de reglas de comportamiento, políticamente consensadas entre las fuerzas políticas, lo cual las convierte en una fuerza centrípeta que coadyuva a la eliminación de corrientes fragmentarias, a la vez que es el único instrumento que actúa como una especie de código de conducta, y que tiene la virtud de poseer medios semicoactivos.

En síntesis, diremos que la competencia es la estructura de normas y reglas que garantizan la funcionalidad del sistema electoral, enmarcado por valores de contenido que coadyuvan a la realización de procesos electorales entre adversarios políticos, lo cual evita potencialmente la excesiva fragmentación, así como la ruptura de dicho sistema.

En el análisis presente, la competencia la vamos a identificar dentro de dos rubros: a) las condiciones sistémicas de la competencia, y b) las condiciones directas de la competencia.

Competitividad implica el resultado de la puesta en marcha de las condiciones de la competencia, por ello califica y mide la capacidad del uso de los medios y recursos que el sistema electoral de un país pone a disposición de los actores políticos; es decir, la competitividad representa la movilización más o menos racional de las condiciones de la competencia.

En la perspectiva antes descrita, la competitividad siempre será un acto ex post, pues su evaluación se mide en función de sus resultados; es decir, de hechos consumados. Así, la competitividad mide esencialmente dos cosas: de un lado, la capacidad de lucha política de los partidos, y del otro, la vigencia de la competencia como estructura.

La competitividad de un partido, por lo tanto, depende en buena medida de la estructura de competencia, asi como de la destreza, cultura y racionalidad en que son puestos en marcha los recursos y medios de que disponen durante un proceso electoral, La competitividad es, en cierta medida, el resultado que produce aquella famosa caja negra de los procesos sistémicos, y la cual no oculta en su interior ningún misterio, salvo la multiplicidad de combinaciones de dichos recursos y medios que mecanizan cada uno de los operadores bajo su responsabilidad.

Para establecer más la diferencia específica entre la competencia y la competitividad, la primera es originaria y relativamente estable, mientras que la segunda es dinámica, de modo que puede, incluso, conmover y adjetivar a la primera, de tal forma que

[...] un sistema sigue siendo competitivo estructuralmente, mientras las políticas se vean control adas por la conciencia de que podría entrar en el mercado un nuevo competidor, y que grandes sectores del público podrían modificar sus lealtades. (Sartori. 1987:264) 


\section{LAS CONDICIONES DE LA COMPETENCIA EN LA ACTUAL LEGISLACIÓN ELECTORAL}

En consecuencia a lo que se ha dicho hasta el momento, resulta claro que no podemos abordar el tema de la competitividad de los partidos políticos en Baja Califomia durante el proceso electoral del 6 de julio de 1997, sin que podamos tocar el problema de la identificación, por lo menos, de la estructura de la competencia.

A este respecto, rescataríamos no solamente el tema del financiamiento, así como la utilización de los medios de comunicación masiva, sino otros elementos que en su conjunto constituyen la estructura normativa de la competencia.

Sin embargo, vale la pena, para el caso de México, hacemos quizá la siguiente pregunta de cara a las reformas recientes: ¿cuáles fueron las razones que alentaron a las fuerzas políticas en la actual reforma electoral?

Garantizar el principio de equidad en las condiciones de la competencia, de modo tal que el financiamiento y el acceso a los medios de comunicación de esas organizaciones se convierta en un elemento que fomente una mejor competencia política. (Becerra, 1997:106, 107)

A simple vista parecía una lucha y una propuesta de corto alcance, por los limitados efectos que aparentemente ofrecía una propuesta de estas características. Sin embargo, dada la profunda penuria de los partidos de oposición a lo largo del tiempo en que operó nuestro sistema de partido hegemónico, y que contrastó notoriamente con la abundancia de recursos de que hacía gala el partido oficial (PRI), la lucha de las organizaciones políticas por establecer en la legislación mexicana la vigencia del principio de equidad, se constituy6 en un asunto nuclear en cualquier coyuntura en la cual estuviera en debate la modificación de las reglas de la competencia electoral.

Tal reclamo era válido por dos razones: primero, por su impacto en el desenvolvimiento y futuro del propio sistema de partidos, pues como señala Heinrich Triepel, los temas del funanciamiento público, así como los del uso de los medios de comunicación masiva, constituyen una muestra típica de la madurez de un sistema democrático; y segundo, porque el avance y el enriquecimiento de las condiciones de la competencia impulsan el desarrollo, las posibilidades de expansión y conquista de mayores clientelas para cada uno de los partidos, además de ampliar la potencialidad de convencimiento a través de los medios y pluralizan las orientaciones políticas. Esto tiene efectos tanto en las unidades políticas como en los ciudadanos. 
En la historia política de México, el asunto de los elementos con que deben contar los partidos, tales como recursos financieros, acceso y utilización de los medios de comunicación masiva para la difusión de sus ideas y propagandas, consiste en prerrogativas muy limitadas, e incluso imprecisas, pues todavía en la legislación electoral de 1977-1978, apenas se menciona el derecho de acceso de elementos para la realización de sus actividades partidarias y electorales. Por ello, recupera interés señalar que no es, sino a partir del Código federal de instituciones y procedimientos electorales, cuando se da un gran salto en dicha materia; ésta ha sido enriquecida, sin duda, durante el periodo intenso de reformas electorales (1993-1996), hasta culminar con un sistema de medios y prerrogativas (SMP) hacia los partidos políticos notoriamente avanzado, incluso similar a los que funcionan en otros paises de democracia consistente y altamente competitiva.

En nuestro sistema electoral, tal avance sín duda está ligado a los dos fines que ya se han señalado; es decir, la urgente necesidad de dar saltos importantes en la maduración de la democracia electoral, y como diria un autor, "es una consecuencia de la obligación de reactivar el vínculo entre los electos y los electores, mediante la periódica movilización electoral". (Lagroye, 1994:361)

Para efecto de nuestro estudio, hemos partido del análisis del Código federal de instituciones y procedimientos electorales (Cofipe), con el fin de determinar, de acuerdo con éste, la estructura de la competencia, a la cual vamos a denominar condiciones de la competencia; ésta, a su vez, y derivado de un intento de sistematización simple, la dividimos en: a) condiciones sistémicas de la competencia, y b) condiciones directas de la competencia.

Las condiciones sistémicas de la competencia, como podemos observar en el cuadro 1, contienen todas aquellas normas, reglas y valores que resultan imprescindibles e insustituibles - salvo por otras más completas y desarrolladas - que determinan la naturaleza competitiva de nuestro sistema electoral. Contiene la impregnación de los valores de contenido que solidifican los actos de consenso de las fuerzas políticas organizadas, lo cual to vuelve vigente $\mathrm{y}$ aplicable, incluso cuando es necesaria la reacción coactiva por parte de las autoridades electorales.

Las normas contenidas en las condiciones sistémicas constituyen el piso común, así como el marco de actuación de todas las fuerzas políticas organizadas, debido a tres principios sustanciales: igualdad, libertad y equidad.

Los principios antes señalados, impregnan los contenidos de los actores y de los actos fundamentales del sistema electoral mexicano: a) partidos 


\section{CUADRO 1. Las condiciones de la competencia en la legislación electoral.}

Cofipe Condiciones sistémicas Cofipe Condiciones sistémicas

Art. 4 Votar en las elecciones constituye un derecho y una obligación del ciudadano.

El voto es universal, libre, secreto, directo, personal e intransferible.

Art. 5 Es derecho de los ciudadanos mexicanos constituir partidos políticos nacionales y agrupaciones políticas, $y$ afiliarse a ellos individual y libremente. Es derecho exclusivo de los ciudadanos mexicanos participar como observadores de los actos de preparación y desarrollo del proceso electoral, así como de los que se lleven a cabo el dia de la jornada electoral.

Art. 7 La observación completa de los requisitos para ser diputado federal o senador.

Art. 24 Para que una organización pueda ser registrada como partido político nacional deberá cumplir los siguientes requisitos:

a) Formular una declaración y, en congruencia con ellos, su programa de acción y los estatutos que nomen su actividad.

b) Contar con 3000 afiliados en por lo menos 10 entidades federativas, o bien. 300 afiliados en por lo menos 100 distritos electorales uninominales.

Además, deberá cumplir los requisitos procedimentales establecidos en el

Cofipe

Art. 36 Se consideran condiciones sistémicas el apartado de derechos asignados a los partidos políticos como:

a) Participar en la preparación, desarrollo y vigilancia del proceso electoral.

b) Postular candidatos en las elecciones federales.

c) Formar frentes y coaliciones, así como fusionarse.

d) Nombrar representantes ante los órganos del Instituto Federal Electoral.
Art. 38 Se consideran condiciones sistémicas el apartado de obligaciones de los partidos políticos como:

a) Conducir sus actitividades dentro de los cauces legales, respetando la libre participación política de los demás partidos políticos y los derechos de los ciudadanos:

b) Abstenerse de recurrir a la violencia $y$ a cualquier acto que tenga por objeto o resultado alterar el orden público y perturbar el goce de las garantías. c) Mantener el mínimo de afiliados en las entidades federativas o distritos electorales.

d) Mantener en funcionamiento efectivo a sus ónganos estatutarios.

e) Actuar y conducirse sin ligas de dependencia o subordinación con partidos políticos, personas físicas o morales extranjeras, organismos o entidades internacionales y de ministros de culto.

f) Abstenerse de cualquier expresión que implique dialriba, calumnia, infamia, injuna, difarnación o que denigre a los ciudadanos, a las instituciones públicas o a otros partidos políticos y sus candidatos.

g) Abstenerse de realizar afiliaciones colectivas de ciudadanos.

Art. 69 Se consideran condiciones sistémicas la suscripción de algunos de los fines de la institución.

a) Integración del registro federal de electores.

b) El aseguramiento a los ciudadanos del ejercicio de los derechos políticoelectorales.

c) Velar por la autenticidad y efectividad del sufragio.

d) La observancia de los principios de certeza, legalídad, independencia imparcialidad y objetividad. 


\section{CUADRO 1. (Continuación).}

$\begin{array}{lll}\text { Cofipe } & \text { Condiciones sistémicas } & \text { Cofipe }\end{array}$

Art. 70 Es una condición sistémica el carácter autónomo, permanente, la independencia en sus decisiones y funcionamiento, así como contar con personalidad jurídica y patrimonio propios, cualidades éstas del Instituto Federal Electoral.

Art. 175 Corresponde exclusivamente a los partidos politicos nacionales, el derecho de solicitar el registro de candidatos a cargos de elección popular.

Art. 269 Los partidos políticos podtán ser sancionados por los siguientes motivos: a) Incumplan las obligaciones que sefiala la ley de la matería.

b) Acepten donativos o aportaciones económicas de las personas o entidades que no estèn expresamente facultadas para ello.

c) Acepten donativos o aportaciones económicas superiores a los límites sefialados en la ley.

d) Sobrepasen durante la campaña electoral los topes a los gastos fijados

\section{Ley general del sistema de medios de impugnación}

Ant. 3 El sistema de medios de impugnación tiene por objelo garantizar:

a) Que todos los actos y resoluciones de las autoridades electorales se sujeten invariablemente a los principios de constitucionalidad y legalidad; este sistema de impugnación se intega por los recursos de revisión, el recurso de apelación, el juicio de inconformidad y el recurso de reconsideración, el juicio para la protección de los derechos político-electorales del ciudadano y el juicio de revisión constitucional electoral, para garantizar la constitucionalidad de actos o resoluciones de las autoridades locales en los procesos electorales de las entidades federativas. 
políticos, b) ciudadanos, y c) autoridades electorales. Constituyen, en suma, los derechos, obligaciones y sanciones que en forma equitativa les corresponde a cada una de las unidades políticas legalmente reconocidas, de manera similar que a los actores restantes del proceso electoral. Pero, además de incluir los derechos, obligaciones y sanciones, contiene las características distintivas que convierten al sistema electoral en semicompetitivo, en transición hacia uno competitivo, y que permiten ubicarlo en la escala de los modelos, que va de los competitivos, pasa por los semicompetitivos o autoritarios, y en el extremo, a los totalitarios o no competitivos.

La segunda división de las condiciones de la competencia, la bemos denominado condiciones directas, y en esta parte están contenidos los siguientes aspectos: a) financiamiento, b) radio y televisión, c) régimen fiscal, y d) franquicias postales y telegráficas.

Las condiciones directas tienen la virtud de que su utilización puede tener efectos notorios en la competencia, pues implica, de hecho, aplicar una serie de factores externos al partido político con el objeto de potenciar su capacidad de cobertura, penetración y oferta hacia el mercado político (cuadro 2).

Las condiciones directas constituyen el mayor logro del sistema electoral mexicano en la procuración del cumplimiento de tres objetivos: a) desemplazar el sistema de privilegios que tradicionalmente, durante la etapa del sistema de partido hegemónico, disfrutó el Partido Revolucionario Institucional (PRI); b) establecer condiciones de equidad relativa en materia de recursos financieros y uso de medios, de tal forma que éstos redujeran las diferencias acumuladas a favor del PRI y en contra de los partidos de oposición; y c) prever que en el futuro no se contituyeran condiciones de inequidad y privilegios, que pudieran conceder ventajas ilegales y de poca claridad hacia un partido político en contra de los intereses de los demás.

Los factores contenidos en este rubro tienen un significado en términos valorativos y de principios, pues denotan un vuelco radical que va de un sistema de inequidad, imprecisión, desigual e ilegal, hacia otro en el cual el trato igualitario y equitativo son algunos de sus símbolos; sin embargo, aunque éste proporciona los recursos y medios a través de los cuales los partidos políticos, en términos generales, tendrian similares posibilidades para su crecimiento y desarrollo, la suma de las condiciones sistémicas y las condiciones directas, no garantizan que el sistema electoral sea absolutamente competitivo. A propósito de ello, nosotros pensamos también que "en la práctica la perfecta competencia electoral representa un objetivo tan irreal como sucede con la libertad absoluta del elector" (Hermet, 1997: 24). No obstante, el logro es notable. 


\section{LA COMPETITIVIDAD PARTIDARIA DURANTE EL PROCESO ELECTORAL DE 1997}

Una vez que hemos establecido la importancia de medir la competitividad de los partidos políticos, abora quisiéramos avanzar en la medición, precisamente, de su efectividad, para lo cual tomaremos como base los números que arrojó la elección federal del 6 de julio de 1997, en lo que respecta a diputados de mayoría relativa en Baja California.

Antes de inferir cualquier conjetura derivada del análisis de dicha efectividad, conviene realizar algunas acotaciones introductorias a este respecto:

Una que resulta interesante, y que pudiera servir de llamado precautorio para los analistas del comportamiento electoral, así como de la fuerza de los partidos políticos en Baja California, es que el estado ha mostrado un comportamiento sumamente irregular en relación con la decisión ciudadana, pues podemos observar, tentativamente - ya que no es éste el objetivo del trabajo - tres periodos de conducta electoral en el reciente pasado histórico (1970-1997): uno que va de 1970 a 1989, el cual lo podemos caracterizar por un alto grado de abstención (nunca menor a $40 \%$ ); a su vez, una notoria declinación de la votación a favor del PRI, lo cual se contrasta con una alza del Partido Acción Nacional, el que incluso, a partir de 1983, inicia su periodo más benéfico, culminando con la adjudicación de la gubematura del estado en 1989. El segundo periodo, de 1991 a 1994, se caracteriza por una notoria alza de la participación ciudadana con números relativos de $78 \%$, y en el que el Partido Revolucionario Institucional registró triunfos en elecciones federales y locales, obteniendo, sin embargo, el Partido Accion Nacional mejores resultados en las elecciones, en las cuales se disputó el poder local. Y el tercer periodo, que parece baberse iniciado en 1995 con elecciones locales, y cuyas tendencias indican que se han confirmado en la elección de 1997, se caracteriza por un notorio decaimiento de la participación ciudadana, pues en 1995 se obtiene apenas 62\%, mientras que la abstención es de $38 \%$ (los votos y el poder local se dividen fundamentalmente entre el PRI y el PAN, con ventaja para este último, pues retiene un bastión relevante, que es la gubernatura del estado). Una segunda fase de este periodo lo constituye el resultado de la eleccion de 1997, en la cual nuestro estado se conduce de la siguiente manera: se registra una participación general de $51 \%$, y por lo tanto la abstención alcanza 49\%, Por otro lado, el resultado en términos de poder, muestra una derrota notoria del PRI, pues pierde en cinco de seis distritos electorales uninominales, adjudicándose en contrapartida el PAN los cinco distritos antes señalados. 


\section{CUADRO 2. Las condiciones de la competencia en la legislación electoral}

\section{Cofipe}

Financiamiento

\section{Condiciones directas}

El financiamiento de los partidos políticos tendrá las siguientes modalidades:

a)Financiarniento público, que prevalecerá sobre los otros tipos de financiamiento.

b) Financiamiento por la militancia.

c) Financiamiento de simpatizantes.

d) Autofinanciamiento

e) Fina nciamiento por rendimientos financieros, fondos y fideicomisos.

Los partidos políticos tendrán derecho al financiamiento público de sus actividades conforme a lo siguiente:

a) Para el sostenimiento de actividades ordinarias permanentes:

Este se distribuirá, una vez determinado su monto, de la siguiente manera: $30 \%$ se entregará en forma iguatitaria a los partidos políticos con representación en el congreso. El $70 \%$ restante se distribuye de acuerdo con la fuerza electoral obtenida en la elección de diputados inmediata anterior.

b) Para gastos de campa ña:

A cada partido se le otorgará para estos gastos, un monto equivalente al financiamiento público que para el sostenimiento de sus actividades ondinarias permanentes le corresponda en ese año.

c) Por actividades especificas como entidades de interés público: En este caso el apoyo financiero no podrá ser mayor al $75 \%$ antal de los gastos que los partidos hayan erogado por esle concepto el año inmediato anlerior.
Los partidos que hayan obtenido su registro con fecha posterior a la última elecciôn, se les otorgará financiamiento público de la siguiente manera:

Para el sostenimiento de sus actividades ordinarias permanentes se les otorgará

$2 \%$ del monto total que les correspondió a los demás partidos; corresponderá una cantidad adicional igual para gastos de campaña.

De manera similar, se les otorgará el fina nciamiento público por sus actividades especificas:

El financiamiento por la militancía:

Éste estará confornado por las cuotas obligatorias ondinarias y extraordinarias de sus afiliados, las de sus organizaciones sociales y por las cuotas de los candidatos para sus campa ñas.

El linanciamiento de simpatizantes estara conformado por las aportaciones o donativos, en dinero o en especie, hechas por las personas físicas o morales mexicanas con residencia en el país; éstos se sujetarán a lo siguiente: las aportaciones anuales de simpatizantes no serán superiores a $10 \%$ del financiamiento público para actividades ordinarias; las aportaciones hechas por persona física o moral, tendrá un límite anual igual a $0.05 \%$ del financiamiento público para actividades ordinarias.

El autofinanciamiento se constituye por los ingresos de los partidos obteridos por sus actividades promocionales como conferencias, espectáculos. juegos y sorteos, eventos culturales y ventas cditoriales.

Para el financiamiento por rendimientos fínancieros, las partidos políticos podrán crear fondos of fideicomisos con su patrimonio. 
CUADRO 2. (Continuación).

Cofipe $\quad$ Radio y Television $\quad$ Condiciones directas $\quad$ Radio y Televisión

Art. 41 Acceso en forma permanente a la radio y televisión.

Art. 42. Los partidos políticos, al cjercer sus prerrogativas en radio y televisión. deberán difundir sus principios ideológicos, programas de acción y plataformas electorales.

Ait 44 Del tiempo total que le corresponde al Estado en las frecuencias de radio y en los canales de televisión, cada partido politico disfrutará de 15 minu los mensuales en cada uno de estos nedios de comunicación. Los partidos políticos tendrán derecho a participar conjunta mente en un programa especial que establecerí y coordinará la dirección ejecutiva de prenogativas y partidos politicos, para ser transmitido por radio y televisión dos veces al mes.
Art. 47 Los partidos políticos, durante las campañas electorales, a fin de difundir sus candidaturas, independientemente del tiempo previsto en el art. 44 de este código, tendrán derecho a lo siguiente:

a) Para todos los partidos y cuando se elija presidente de la república, el total de transmisión será de 250 horas en radio y de 200 en televisión b) Cuando se elija sólo a integrantes del congreso, el tiempo de transmisión será $50 \%$ de lo previsto en el inciso anterior.

c) Durante el tiempo de las campa hias clectorales, adicionalmente, se pon drán a disposición de los partidos políticos mensualmentè, hasta 10000 promocionales de radio y 400 en televisión con diración de 20 segundos

Para los partidos sin representación en el congreso, se les asigna $4 \%$ del total de las transmisiones previstas en las incisos a) y c); el resto se distribtin entre los partidas con representación de la siguiente manera: $30 \%$ en forma igualitana y $70 \%$ restante en proporción a su fuerza electoral.
Art 5 Los partidos políticos nacionales no son sujetor de los impuestos y derechos siguientes:

a) Los relacionados con rífas y sorteos, ferias y festivales.

b) Sobre la renta, enajenación de inmuebles, asi como donaciones.

c) Venta de impresos para la difusión de sus principios, programas, estatuios

y en general, para su propaganda, uso de equipos y medios audiovistales.
Art. 53 Los partidos políticos disfrutarin de las franquicias postales y telegráficas dentro del territono nacional, que sean necesarias para el desarrollo de sus actividades. 
En este panorama de datos y cifras, lo que podemos rescatar a propósito de nuestra indagación, es una serie de conjeturas y preguntas:

- Primera conjetura: El sistema electoral en Baja California se ha caracterizado por un comportamiento electoral inestable e irregular; sin embargo, parece ser que por algún tiempo tendremos que acostumbrarnos a que la disputa por el poder se realizará entre dos partidos significantes, a saber: el PRI y el PAN.

- Segunda conjetura: En atención a las dualidades de nuestra realidad nacional, en la cual cohabitan dos o más sistemas, siendo incluso contradictorios entre sí, para el caso de nuestro estado, el tránsito hacia un modelo de partidos que va del begemónico predominante hacia uno de pluralismo moderado, no se ha operado con claridad, y aún persiste una especie de bipartidismo, en el cual sólo dos fuerzas se conducen como partidos predominantes, y cuya totalidad del poder se comparten, incluso, alternativamente.

Una vez planteadas las anteriores conjeturas, vale la pena elaborar las siguientes preguntas;

¿Qué tan eficaces han sido los partidos políticos en la última elección? Y precisamente, ¿cuáles partidos políticos ban sido eficaces?

Sin duda, como ya se ha senalado, el comportamiento errático electoral manifestadoen el estado, nos inclina a pensar que una parte de la responsabilidad corre por cuenta de los partidos políticos quienes, por conducto de una serie de medios y recursos (condiciones sistémicas y condiciones directas de la competencia) puestos a operar, pretenden movilizar al electorado a fin de obtener el mayor número de ellos a su favor, dado que su mutación en escaños indica, finalmente, no sólo su fuerza electoral, sino también su fuerza parlamentaria. Aceptando que "su capacidad de penetración en los diversos sectores de la sociedad, su aptitud competitiva, y su efectividad para trocar votos por puestos de representación popular", todo ello aunado a "sus definiciones ideológicas, sus planteamientos programáticos [...] la presencia y popularidad de sus dirigentes y candidatos" (Valdez, 1995:31), constituyen una condición de eficacia, diremos que lo que pretendemos en esta parte del análisis, es determinar, a través de distintos indicadores, la interacción competitiva que existe entre los partidos políticos significantes del sistema electoral de Baja California.

\section{Competitividad simple}

Este primer indicador de competitividad se refiere a una cuantificacion simple de puestos adjudicados por los diferentes partidos, gracias a los votos obtenidos en la contienda electoral. 
Para el caso de Baja Califomia, y en el cual estuvieron en disputa seis distritos electorales uninominales ( 01 y 02 corresponden a Mexicali; 03 a Ensenada, Rosarito y Tecate, y 04,05 y 06 a Tijuana), en el cuadro 3 puede observarse que el Partido Acción Nacional obtuvo cinco diputaciones de mayoría relativa, lo cual equivale a $83.3 \%$, obteniendo, por ello, los distritos 02 de Mexicali, 04, 05 y 06 de Tijuana, y el 03 con cabecera en Ensenada. Por su parte, el Partido Revolucionario Institucional obtuvo una diputación, to cual equivale a $16.7 \%$, correspondiente al distrito 01 de Mexicali. Un señalamiento que vale la pena anotar, es que los demás partidos no obtuvieron ningún triunfo en alguno de los distritos electorales federales.

\section{CUADRO 3. Triunfos obtenidos por cada partido político.}

\begin{tabular}{cccc}
\hline Partido & Distritos & $\%$ & Observaciones \\
\hline PAN & 5 & 83.3 & $\begin{array}{c}\text { Distritos 02, 03, 04, } \\
05 \text { y 06 }\end{array}$ \\
PRI & 1 & 16.7 & Distrito 01 \\
Total & 6 & 100.0 & \\
\hline
\end{tabular}

FUENTE: Elaborado por el autor con base en información del IFE.

Una inferencia notable respecto a este indicador, es que existe una evidente falta de congruencia entre los votos obtenidos y los puestos de representación conquistados, ya que el PAN, habiendo obtenido $41,8 \%$ de la votación, se le adjudicó $83.3 \%$ de los puestos de elección disputados. De manera similar, la asimetría se presenta en el PRI, pues obteniendo $34.5 \%$ de votación a favor, se le adjudic $616.7 \%$ de los puestos de elección en dísputa. La situación más notoria es para el PRD, el cual obteniendo 13\% de la votación a su favor, no le significó ningún puesto de representación. La explicación a este respecto, es que en los sistemas de mayoría relativa existen franjas territoriales en los que esta inequidad se manifiesta con mayor rigor: sin embargo, ello se subsana, en mayor o menor medida, aplicando la regla de representación proporcional a nivel de circunscripción y a nivel nacional, a través de la cual los partidos obtienen el número de puestos en una proporción cercana a la cantidad de votos nacionales obtenidos. 
Otra inferencia derivada de este indicador, es que su resultado refleja un alejamiento sustancial del modelo de pluralismo moderado para colocarse en el tipo de sistema bipartidista, pues si recordamos el resultado inmediato pasado durante el proceso de 1994, el PRI fue el partido que obtuvo el $100 \%$ de los puestos de elección popular; es decir, triunfo en los seis distritos electorales uninominales.

Como podemos observar, la disputa por los votos, así como por los puestos, se realizó entre dos partidos políticos, pues los partidos restantes no obtuvieron ninguno de ellos, debido a su baja votacion.

\section{Margen de victoria del PAN con respecto del PRI (mv)}

Se trata de una medida que expresa, en porcentajes, la diferencia de votos entre el primer y segundo lugares en la contienda por las diputaciones de mayoria relativa en los distritos electorales en que se divide la nación. (Valdez, 1995:33)

Algunas de las características de este indicador, es que mide la competitividad y eficacia electoral de dos o tres partidos; sobre todo para el caso de nuestro país, pues tradicionalmente, $y$ aun en épocas recientes, es un partido el que obtiene una mayor cantidad de los puestos de diputados (el PAN para el caso de Baja California en la reciente elección, siguiéndole de cerca sólo el PRI), un segundo el que obtiene una segunda mayoría y, excepcionalmente, un tercero que puede obtener una cantidad también significativa de puestos electorales. En términos generales, el margen de victoria nos permite determinar la distancia electoral entre el partido que obtiene mayor éxito y su más cercano seguidor.

De alguna manera, también este indicador es importante, pues no solo nos permite medir la eficacia de los partidos para obtener más o menos votos, sino que en términos generales nos puede asomar a la calificación del sistema electoral en su conjunto, pues como señala Valdez Zurita, cuando el margen de victoria es muy alto a favor de un partido, quiere decir que en este proceso electoral, por lo menos, fue mucho menor la competitividad del sistema en su conjunto.

De lo anterior es necesario rescatar algo que ya señalamos, en el sentiđo de que la competitividad es dinámica, por lo que darle seguimiento a este indicador, también nos puede mostrar, precisamente, la manera en que alguno o algunos partidos políticos, durante cierto tiempo, han recuperado o adquirido cierta eficacia, mostrándose más o menos competitivos en uno o varios distritos electorales. 
Por lo que tóca a los resultados recientes, como observamos en el cuadro 3, el margen de victoria (mv) se descubrió mediante la comparación solamente de dos partidos políticos: el PAN y el PRI, y dado que en este caso el PAN obtuvo cinco de los seis distritos electorales disputados, la relación será PAN-PRI.

Si tomamos arbitrariamente la decisión metodológica de establecer que el margen menor a $10 \%$ significa los distritos electorales que fueron altamente competitivos, los de un margen comprendidos entre más de $10 \%$ y hasta $30 \%$ como los medianamente competitivos, y los porcentajes mayores de $30 \%$ a aquellos distritos no competitivos, podemos inferir lo siguiente:

- Que solamente el distrito 01, con cabecera en Mexicali, cuyo triunfo logró el PRI, puede considerarse competitivo, precisamente porque el partido opositor en este distrito, el PAN, obtuvo una votación que significó $9.3 \%$ menos que el partido triunfador (cuadro 4 ).

\section{CUADRO 4. Margen de victoria del PAN con respecto del PRI, por distritos en elección de 1997.}

\begin{tabular}{ccc}
\hline Margen \% & Distritos & Observaciones \\
\hline Menos de 10 & 1 & Distrito 01* \\
Entre 10 y 30 & 3 & Distritos 02, 03 y 06 \\
Más de 30 & 2 & Distritos 04 y 05 \\
Total & 6 &
\end{tabular}

* En este distrito el margen de victoria es a favor del PRI.

FUENTE: Elaborado por el autor con base en información del IFE.

- Que tres distritos electorales, el 02, con cabecera en Mexicali, el 03 con cabecera en Ensenada, y el 06 con cabecera en Tijuana, y cuyos triunfos correspondieron al PAN, se pueden considerar semicompetitivos, pues el margen de victoria fue de más de $10 \%$, hasta $30 \%$ en relación con su más cercano opositor: el PRI.

- Que dos distritos, el 04 y el 05, con cabecera en Tijuana, y cuyo triunfo correspondió en ambos al PAN, se consideran no competidos, ya que el margen de victoria obtenido por el PAN sobre el PRI fue de más de $30 \%$. 
- Que en cinco de seis distritos electorales $(02,03,04,05$ y 06$)$ no exístí realmente competitividad entre los partidos políticos, pues el PAN ganó en todos ellos, con márgenes mayores a $10 \%$ con respecto al PRI, su más cercano competidor; es decir, que $83.3 \%$ de los distritos en disputa fueron no competitivos, o a lo mucho, semicompetitivos.

Puede ser importante darle seguimiento a este indicador, pues nos puede brindar algunas pistas claras respecto del comportamiento histórico de los distritos electorales, así como de la dinámica que éstos han demostrado en el transcurso de un lapso determinado. Abundando en ello, podemos identificar un distrito en particular, o varios de ellos, en los cuales ha aumentado, disminuido o permanece inalterable su grado de competitividad, lo cual proporciona datos que pueden alentar alguna estrategia partidaria para modificar tal situación.

Además, el análisis histórico del margen de victoria nos puede informar de la dinámica de la competitividad del sistema electoral en su conjunto, condición sine qua non de un sistema democrático modemo.

\section{Potencia opositora (po)}

$\mathrm{El}$ indicador de potencia opositora (po) permite descubrir cuándo la votación obtenida por el partido triunfador supera los votos obtenidos por el conjunto de los opositores. (Valdez, 1995:35)

Este indicador nos permite identificar varias cosas a la vez:

- Los distritos en los cuales, a consecuencia de una baja competitividad de la oposición en su conjunto, permite que el partido ganador obtenga puestos con mayoría absoluta; es decir, con más de $50 \%$ de los votos emitidos en la elección de diputados de mayoría relativa.

- Descubrir el momento en que, debido a una evolución o estancamiento de dicho indicador, el sistema electoral permanece o avanza de uno de carácter hegemónico o predominante, a otro democrático competitivo.

- Detectar, mediante el análisis histórico, las zonas o distritos de influencia en las cuales, como resultado de múltiples factores de competencia así como ambientales, existe una reincidencia en los números electorales, de tal forma que un partido permanece en dichas zonas o distritos obteniendo triunfos de mayoría absoluta; es decir, a través de ello es posible obtener una geografía de la influencia política por la vía electoral.

En un sistema en transición, como supuestamente es el nuestro, lo ideal es que exista una traducción de aquélla en términos electorales-pragmáti$\cos$, y que la mayoría de los distritos transiten de triunfos por mayoría 
absolutả de un partido a triunfos de mayoria relativa, y además llegar a distritos disputados, en los cuales sea innecesario, por el poco margen de victoria, elaborar el indicador de potencia opositora.

En términos generales, para obtener el indicador de potencia opositora, se contabilizan los distritos, y si la votación alcanzada por un partido supera a la suma de los partidos opositores, es decir, que el triunfo lo logró con más de $50 \%$, se considera mayoria absoluta. Cuando la comparación de dichos resultados nos indican que el partido ganador lo logró con una cantidad igual o menor a la suma de los opositores, es decir, con $50 \%$ o menos, estamos ante un triunfo con mayoria relativa.

Para el caso que nos ocupa, como se puede observar en el cuadro 5 , de los distritos disputados, tres de ellos (que significan el $50 \%$ de distritos), la potencia opositora se ubica en el margen de 1.0 hasta 1.1 ; es decir, tres distritos registraron triunfos de mayoría relativa, que va de $50 \%$ a menos en cada uno de ellos, lo que denota una potencia electoral de la oposición muy baja (en este caso, de todos los partidos sumados, con excepción del PAN, que fue el partido triunfador precisamente en los distritos indicados: 03,04 y 05 ).

CUADRO 5. Potencia opositora en el proceso electoral de 1997.

\begin{tabular}{lccc}
\hline Margen & Distritos & $\%$ & Observaciones \\
\hline De 1.0 hasta 1.1 & 3 & 50 & $\begin{array}{c}\text { Distritos 02, 04 y 05 } \\
\text { todos ellos ganados } \\
\text { por el PAN }\end{array}$ \\
De 1.2 hasta 1.5 & 3 & 50 & $\begin{array}{c}\text { Distrito 01 ganado } \\
\text { por el PRI, y 03 y 06 } \\
\text { ganados por el PAN }\end{array}$ \\
Total & 6 & 100 & \\
\hline
\end{tabular}

FUENTE: Elaborado por el autor con base en información del IFE.

Asimismo, existen otros tres distritos electorales (que representan $50 \%$ del total), en los cuales la potencia opositora va de 1.2 hasta 1.5 , lo cual significa que los partidos triunfadores lograron el triunfo con mayoría relativa, pero con un porcentaje menor a $50 \%$ de la votación total, lo cual muestra que en dichos distritos existe mayor presencia competitiva de otro o de otros partidos políticos. 
El análisis específico de los resultados de la potencia opositora nos sugiere que:

- El signo de la potencia opositora ha cambiado drásticamente, pues en la elección federal de 1994, el Partido Acción Nacional constituía una parte de dichas potencias; sin embargo, en el actual proceso, este partido se ba convertido en el referente para medir dicho indicador por ser el partido triunfador en cinco de los seis distritos. En consecuencia, el PRI ha pasado a formar parte de la suma que se adhiere a la potencia opositora.

- Ningún distrito electoral federal fue ganado por mayoría absoluta, pues en el 100\% de los casos, la potencia opositora nunca fue menos de 1.0 .

- Solamente en un distrito -el 04, que representa $16.6 \%$, y que fue ganado por el PAN-, la potencia opositora es igual a 1.0, por lo tanto, en este caso, el partido ganador obtuvo un porcentaje casi o igual a $50 \%$ de la votación válida; es decir, el triunfo es de mayoría relativa, equiparada a la suma de los votos de toda la oposición.

- Se puede observar que existen dos distritos - 02 y el 05 que equivalen a $33.3 \%$, y que fueron ganados por el PAN-, en los cuales la potencia opositora equivale a 1.1. Lo anterior quiere decir que tales distritos fueron ganados por el Partido Accion Nacional por mayoría relativa. pero con números muy cercanos a $50 \%$ de la votación válida; esto es, que en dichos distritos los votos obtenidos por el partido triunfador equivalen a una cantidad cercana a la suma de votos del resto de los partidos políticos, por ello, la potencia opositora en estos distritos es muy baja.

- En tres distritos -el 01 ganado por el PRI, el 03 y el 06 ganados por el PAN y que equivalen a $50 \%$ de los distritos electorales-, la potencia opositora fue de $1.4,1.5$ y 1.2 , respectivamente. Ello quiere decir que los distritos fueron ganados por mayoría relativa, alejados del $50 \%$ de los votos válidos, por lo que se puede considerar que en éstos es donde existe una mayor polémica electoral de la oposición, ya que la sumatoria de toda la oposición representó más que la votacion obtenida por el partido ganador en cada uno de los distritos.

- Los distritos en donde se observa una mayor potencia opositora son el 01 y el 03 , que equivalen a $33.3 \%$, con una po igual a 1.4 y 1.5 , respectivamente, lo que muestra que es en estos distritos donde existe una mayor competitividad relativa de los partidos opositores.

Para finalizar este aspecto, sólo queremos agregar que este indicador tiene el mérito que, de una sola vez, permite medir la eficacia del partido ganador, así como la competitividad de los partidos perdedores que se suman al rubro de potencia opositora. 


\section{Número de partidos por distrito electoral (np)}

El número efectivo de partidos nos muestra el grado de fragmentación de un sistema de partidos cuya expresión final es la participación de cada partido en la conformación de la cámara legislativa. (Lijphart. 1995:114-116).

Respecto a este indicador, conviene rescatar el apunte que hace Sartori relativo a cuáles partidos cuentan; y a ello habría que contestar, como lo hace en uno de sus textos: cuantitativamente, estableciendo un umbral de votos a su favor, los cuales se traducen en escaños en la Cámara de Diputados, y que le adjudica a dicho partido la capacidad de formar coalición de gobierno con otro u otros partidos; cualitativamente, utilizando su capacidad de chantaje; es decir, influyendo en las condiciones y en la práctica de la competencia, de tal modo que los otros partidos habrán de modificar y/o ajustar su conducta durante las campañas electorales. (Sartori, 1987:57)

Por ello, resulta conveniente poder determinar el número de partidos que estuvieron presentes durante un proceso electoral, ya que permite medir la capacidad de operación de los mismos, pues si insistimos en los dos significados ya descritos, la cuantificación electoral de cada partido se traduce en una medida de eficacia mayor o menor para compartir el poder, asi como también para impactar al propio sistema electoral.

El paso de un sistema de partido hegemónico o predominante a otro democrático competitivo, no se dará mientras sus actores políticos principales no disputen realmente cada uno de los centros de poder del sistema político, incluso que esto suceda de manera combativa y con reducidos márgenes de victoria entre los candidatos ganadores y los perdedores.

Los sistemas de democracia competitiva se edifican sobre la base de una distribución potencial del poder entre los actores políticos, a la vez que da curso a la apertura y entronizamiento de las diversas orientaciones políticas. Es decir, las consecuencias de una democracia competitiva lienen un efecto primario de carácter cuantitativo: cuántos partidos participaron y qué tanto poder se sujeta a su distribución; pero también tiene un efecto cualitativo: cuáles corrientes de pensamiento y orientaciones políticas se integran en el debate nacional en el seno de las instituciones del país.

Por estas razones es interesante el estudio de este indicador. Para tales efectos, procedemos a inferir algunas observaciones derivadas del cuadro 6, relativas al número de partidos por distritos electorales que estuvieron presentes en el proceso electoral federal analizado. 
CUADRO 6. Número de partidos por distritos electorales.

\begin{tabular}{cccc}
\hline $\begin{array}{c}\text { Número de } \\
\text { partidos }\end{array}$ & Distritos & $\begin{array}{c}\text { En relación } \\
\text { con } 6 \text { distritos } \\
\%\end{array}$ & Observaciones \\
\hline Menos de 3.0 & 2 & 33.3 & Distritos 02 y 06 \\
De 3.0 y más & 4 & 66.7 & Distritos 01, 03, 04 Y 05 \\
Total & 6 & 100.0 & \\
\hline
\end{tabular}

FUENTE: Elaborado por el autor con base en información del IFE.

En términos generales, se puede decir que en la contienda electoral pasada, el número efectivo de partidos fue igual a 3.2 ; es decir, que solamente tres partidos políticos realizaron una disputa real por el poder, lo cual se determinó con base en el logro significativo de los votos obtenido en la elección.

Como se puede observar, de los distritos electorales, en dos de ellos -02 y 06 , que representan $33.3 \%$ - el indicador de número de partido (np) fue menos de 3.0; es decir, que los electores en forma mayoritaria se concentraron en más de dos partidos, sin llegar a constituir nunca tres. Éstos serían, de acuerdo con el indicador en cuestión, los distritos en los cuales se expresa una menor competitividad, debido a la ausencia de partidos competitivos que hubiesen podido canalizar a su favor al electorado.

Existe, por otro lado, el resto de los distritos electorales: $01,03,04$ y 05 , que representan $66.7 \%$, y cuyo número de partido (np) fue de $3.0 \mathrm{y}$ más, lo cual quiere decir que tres o más - aunque siempre menos de cuatro, como veremos en el análisis específico-, fueron los partidos que tuvieron presencia electoral durante el reciente proceso. Lo anterior nos habla precisamente, que estamos ante un proceso electoral de muy baja competitividad, en el cual un reducido número de partidos se disputan la mayoría de los votos emitidos por los ciudadanos.

En la misma línea de ideas, si hacemos una conversión de los votos a escaños, lo que obtendremos es un indicador similar al np. A éste lo podemos denominar número de partidos parlamentarios, y se determina aplicando el mismo criterio, sólo que este indicador sí refleja el número de partidos políticos a los cuales se les adjudico, de acuerdo con el resultado electoral, una cantidad determinada de escaños en la Cámara de 
Diputados. Ahora bien, realizando tal operación, tenemos que el número de partidos parlamentarios (npp) durante el reciente proceso es de 1,4, lo cual quiere decir que la totalidad de los escaños (6) se distribuyeron en gran mayoria a un sólo partído (de seis, el PAN obtuvo cinco). Con excepción del PRI, que logra un escaño, el resto de los partidos políticos, no obstante haber tenido una votación determinada, incluso significativa, como el PRD, no fue suficiente, dado el sistema electoral vigente, para lograr algún escaño.

Si analizamos los datos más específicos del caso Baja Califomia, auxiliándonos del cuadro 6 , podemos inferir lo siguiente:

- Ninguno de los distritos entra en la categoria de partido único, pues en ninguno de los seis distritos, el indicador número de partidos es equivalente a 1.0 .

- Los distritos 02 y 06, ambos ganados por el PAN, son los que registran la menor competitividad partidaria, pues como se puede observar en ambos casos, el np es equivalente a 2.9; es decir, que menos de tres partidos se disputaron la gran mayoría de los votos emitidos por los ciudadanos.

- El distrito 01, ganado por el PRI, y el 04 y 05, ganados por el PAN, muestran que en ellos tres partidos y menos de cuatro se adjudicaron la mayoría de la yotación ciudadana durante este proceso. El distrito 03. con cabecera en Ensenada, y que registró un np equivalente a 3.5, es el de mayor competitividad, con una participación menor a cuatro partidos políticos disputándose la mayoría de la votación ciudadana.

Como hemos podido ver, durante la elección próxima pasada, el sistema electoral de Baja Califomia se muestra con una evidente baja competitividad, pues es un reducido número de partidos el que disputan electoralmente cada uno de los distritos, y la marca general de np, que es de 3.2 , nos habla de un comportamiento de poca eficacia partidaria a nivel estatal. Si bien es cierto que en este proceso han desaparecido los distritos de partido único, cuyo índice de np sea igual a 1.0 o menos, los índices registrados sugieren distritos con un partido predominante, pero nunca de un sistema democrático competitivo.

\section{Expansión del sistema electoral (ese)}

La expansión del sistema electoral refleja la relación que existe entre la dinámica de los electores y la de los partidos políticos participantes en las contiendas electorales, expresada proporcionalmente. (Molinar Horcasitas, 1991:161:164). 
Este indicador utiliza dos cifras correspondientes a dos momentos distintos, ya que la relación se establece entre un momento de partida y otro final. En esta magnitud temporal se ubican las diversas dinámicas de los electores, así como las de los partidos políticos que motivan el análisis.

Aunque este indicador no es utilizado frecuentemente como uno que pueda expresar los grados de competitividad, creemos que bien puede brindarnos datos que ayuden a calificar la dinámica del crecimiento electoral de los diversos partidos políticos. Su derivación comparativa, cuyo referente será siempre la expansión o depresión de los electores (padrón o listado nominal), dará una medida indirecta de la eficacia de los partidos políticos.

Lo ideal es que este indicador refleje una cierta simetría o proporción entre la dinámica de los electores (expansión o depresión del padrón o listado nominal) y la dinámica electoral de los partidos políticos en una de sus manifestaciones. La otra es que a una mayor eficacia de algún o de algunos partidos políticos, deberá corresponder un crecimiento de su acervo electoral, incluso más allá de la proporción en que se manifiesta la dinámica de los electores.

Es necesario enfatizar que también el análisis de la expansión del sistema electoral resulta más adecuado para mediciones longitudinales; es decir, tomar como horizonte de estudio diversos periodos, ya que ello permite determinar la evaluación de ambos fenómenos: la dinámica de los electores y la dinámica de los partidos políticos, para luego establecer una interrelación entre ambos, lo cual da una idea de cierta competitividad entre los actores políticos frente al comportamiento de los nuevos electores.

Resulta conveniente señalar que para el caso que nos ocupa, el de Baja California, los seis distritos electorales federales sufrieron una serie de adecuaciones durante 1996, dado la ejecución de un proyecto de redistritación, lo que implicó alterar el proceso de crecimiento natural de ciudadanos de los ámbitos territoriales distritales, y a este proceso se le adhirió uno de carácter artificial, resultado de la nueva adecuación. Ello implicó que a los distritos 01 y 04 (antes IV y vI, respectivamente) se les redujera significativamente tanto el ámbito territorial como el poblacional, y en consecuencia el de los ciudadanos.

Por su parte, los distritos $02,03,05$ y 06 (antes I, III. V y II, respectivamente) registraron crecimientos en el ámbito territorial, así como en el poblacional, y en consecuencia el de ciudadanos.

No obstante lo anterior, la formula sigue siendo eficiente, ya que ambas dinámicas (la de electores y la de votación de partidos) se establecen en proporción a los crecimientos y depresiones registradas entre sí. 
En términos generales, se puede observar, a partir de los datos contenidos en el cuadro número 7, que el listado nominal en Baja California durante el periodo de 1994-1997 tuvo un crecimiento equivalente a $18.5 \%$. $\mathrm{Y}$ sin embargo, los partidos PAN y PRI obtuvieron, durante el mismo periodo, un crecimiento equivalente a $-9.6 \%$ y $-83.1 \%$ respectivamente, mientras que el PRD obtuvo un índice de crecimiento equivalente $a+33.2 \%$, lo cual indica que el PRI es el que tiene el más bajo índice de eficacia en la función de orientar los votos a su favor. De manera similar, el PRD aparecería como el partido más eficiente, pues es el que mostró mayor capacidad para aumentar su acervo electoral.

Por otra parte, se puede observar que el PAN demuestra que en tres distritos $(01,03$ y 04$)$ no pudo aumentar su acervo electoral, pues en ellos tuvo porcentajes de crecimiento negativos. A su vez, en los tres distritos restantes $(02,05$ y 06$)$ sí registro índices de crecimiento positivo. En términos generales, ello indica una efectividad relativa, lo cual se corrabora al hacer el análisis comparativo con la dinámica del listado nominal respectivo.

Por su parte, el PRI muestra un crecimiento negativo en los seis distritos electorales, lo que significa, de entrada, una clara falta de eficacia para dinamizar su capacidad electoral.

Finalmente, el Partido de la Revolución Democrática registra que sólo en un distrito electoral (el 04) obtuvo un crecimiento negativo, mientras que en el resto de ellos $(01,02,03,05$ y 06$)$, su crecimiento electoral registró números positivos, lo cual, de acuerdo con el significado de este indicador, el PRD se constituye como el de mayor eficacia relativa, situación que habremos de constatar cuando se realice el análisis especifico de cada uno de los distritos.

El estudio de los datos concretos del cuadro 7 nos refleja lo siguiente:

- El distrito 01 (antes IV), con cabecera en Mexicali, registró un descenso en su listado nominal de 1994 a 1997 , equivalente a $-17.2 \%$. Ello obedece a que este distrito transfirio hacia el distrito 02 (antes $n$ la cantidad de 80 secciones, que representaron 64197 ciudadanos. El comportamiento ideal hubiera sido que, gracias a este indicador, los partidos hubiesen mostrado una dinámica similar o proporcional al del listado nominal; sin embargo, como puede observarse, el PAN y el PRI tuvieron depresiones muy significativas equivalentes a $231 \%$ mayor que la depresión del listado nominal (esto es en el caso del PAN). Para el PRI, el indicador es de $576.7 \%$ mayor que la depresión del listado nominal. Sin embargo, el PRD es el que acusa una mayor expansión positiva de $8.0 \%$, no obstante la depresión del listado nominal; es decir, en este caso, el PRD es el partido que logra una mayor competitividad histórica, tomando como referencia la dinámica del listado nominal. 


\section{CUADRO 7. Indicador de competitividad: expansión del sistema electoral.}

\begin{tabular}{cccccc}
\hline Distritos & Periodo & $\begin{array}{l}\text { Crecimiento } \\
\text { de listado } \\
\text { nominal }\end{array}$ & $\begin{array}{l}\text { Crecimiento } \\
\text { del PAN } \% \\
\text { (votos) }\end{array}$ & $\begin{array}{l}\text { Crecimiento } \\
\text { del PRI \% } \\
\text { (votos) }\end{array}$ & $\begin{array}{l}\text { Crecimiento } \\
\text { del PRD \% } \\
\text { (votos) }\end{array}$ \\
\hline & & & & & \\
01 & $1994-1997$ & -17.2 & -57.1 & -116.4 & 8.0 \\
02 & $1994-1997$ & 67.9 & 51.3 & -36.5 & 105.9 \\
03 & $1994-1997$ & 29.4 & -0.6 & -77.6 & 54.9 \\
04 & $1994-1997$ & -37.0 & -79.3 & -240.0 & -44.3 \\
05 & $1994-1997$ & 68.6 & 19.0 & -31.6 & 90.5 \\
06 & $1994-1997$ & 85.9 & 35.2 & -11.7 & 116.6 \\
Total & $1994-1997$ & 18.5 & -9.6 & -83.1 & 33.2 \\
\hline
\end{tabular}

- El distrito 02 (antes I) con cabecera en Mexicali, mostró un ascenso en su listado nominal en el mismo periodo, equivalente a $67.9 \%$. Ello obedece a una mezcla de factores de crecimiento poblacional, como al hecho de haber recibido del 01 (antes IV), la cantidad de secciones y ciudadanos arriba descritos. Como podemos observar, el PRJ es el único partido que registra una asimetría entre la dinámica del listado nominal y el de su propio comportamiento, obteniendo $-36.5 \%$ de crecimiento electoral en dicho periodo. El Partido Acción Nacional muestra una dinámica acorde proporcionalmente al crecimiento del electorado, con $51.3 \%$ de crecimiento. Mientras que el PRD se muestra como el más competitivo, tomando como referencia la dinámica de los electores, con un crecimiento en dicho periodo de $105.9 \%$.

- El distrito 03 (antes III), con cabecera en Ensenada, registró, en su lista nominal, un crecimiento de $29.4 \%$, derivado de la adhesión del municipio de Rosarito, lo cual implicó la adjudicación de 27 secciones y 21472 nuevos ciudadanos (además del crecimiento natural). El comportamiento muestra que el PAN decreció en $-0.6 \%$ su dinámica electoral durante el periodo; el PRI decreció $-77.6 \%$, mostrando la mayor desproporción entre el listado nominal y su dinámica electoral; y el PRD es el que con $54.9 \%$, muestra el mayor dinamismo electoral en este distrito, pues esta cifra representa $86.7 \%$ más del crecimiento que registró el listado nominal.

- El distrito 04 (antes Vl), con cabecera en Tijuana, obtuvo, en el periodo analizado, un crecimiento en su listado nominal equivalente a $-37 \%$. derivado de una transferencia de secciones equivalente a 130 que realizó 
hacia el distrito 05 , que representaron la adjudicación de 130137 ciudadanos. De manera similar se asignó al distrito 06 (antes II), un total de 20 secciones con 18559 ciudadanos; por su parte, recibe del distrito 05 la cantidad de 27 secciones, con la adhesion de 21615 ciudadanos. En este distrito, los tres partidos analizados (PRI, PAN y PRD) registran crecimientos negativos en su dinámica electoral, incluso todos lo hacen en forma desproporcionada en relación con la dinámica del listado nominal. Sin embargo, por un lado, el PRI es el que registra la mayor depresión con $-240.0 \%$ y que representa $548.6 \%$ más que la dinámica del listado nominal (-37.0\%).

Resulta interesante rescatar que el PAN obtiene una dinámica electoral de $-79.3 \%$, lo cual nos habla de una alta desproporción en relación con la expansión del listado nominal. Por su parte, el PRD es el partido que registra la menor depresión en su dinámica electoral, con $-44.3 \%$, aunque desproporcionalmente a la dinámica del listado nominal.

- El distrito 05 (antes v), obtuvo un crecimiento de su listado nominal equivalente a $68.6 \%$. Ello se derivó de la transferencia que recibió del distrito 04, equivalente a 130 secciones, así como de 130137 ciudadanos; a su vez, cedi6 al 06 la cantidad de 69 secciones, con 51875 electores.

Como podemos observar en este distrito, no obstante el crecimiento de la dinámica de electores, el PRI obtiene una depresión en su dinámica electoral, pues registra un crecimiento de -31.6, lo cual contrasta con el alto crecimiento de electores. Por su parte, el Partido Acción Nacional, si bien obtiene resultados positivos con un crecimiento equivalente a $19.0 \%$, éste noes proporcional en relación con el crecimiento del listado nominal. Mientras que el Partido de la Revolución Democrática es el que demuestra mayor eficacia, pues obtiene un crecimiento en dicho periodo, equivalente a $90.5 \%$, lo cual significa $31.9 \%$ más que el índice de crecimiento del listado nominal.

- El distrito 06 (antes II), con cabecera en Tijuana, es el que registra el mayor crecimiento en su dinámica electoral con $85.9 \%$. Dicho crecimiento tiene su origen al recibir 20 secciones del distrito 04, que incluyeron a 18559 electores. A su vez, tambien recibe del 05 la cantidad de 69 secciones que incluyó a 51875 nuevos ciudadanos. El análisis sobre el impacto que dicho crecimiento tuvo, refleja que el PRI es el partido que mayores efectos negativos registró, con un crecimiento de $-11.7 \%$. El Partido Acción Nacional registró números positivos equivalentes a $35.2 \%$, aunque dichos resultados no reflejan una proporcionalidad en relación con el crecimiento del listado nominal. Finalmente, el Partido de la Revolución Democrática es el que muestra 
mayor efectividad, con un crecimiento de $116.6 \%$, equivalente a un $35 \%$ mayor que el crecimiento del listado nominal.

\section{CONCLUSIONES}

Por todo lo anterior, se puede concluir lo siguiente: Dado que la competitividad constituye, junto con la competencia, el rasgo tal vez más sobresaliente y distintivo de, en primer lugar, la democracia política, y en segundo, del grado de desarrollo de un sistema de partidos políticos, conviene determinar la magnitud de avance y expresion de ambos fenómenos en nuestro país. De aquí que una primera inferencia que podemos sustraer del presente estudio, es que la competencia, conceptuada como el sistema normativo que determina las características y reglas que garantizan la actividad proselitista de los partidos políticos, ha sufrido un irregular itinerario, cuyo curso se ha caracterizado por el reconocimiento variable del rol que juegan los partidos políticos en el sistema electoral mexicano; esto, para nosotros, es una señal ínequívoca del desnivel de las fuerzas políticas insertas en la vida política del país. Por otra parte, cuando las fuerzas políticas son realineadas, precisamente es que las condiciones de la competencia han cambiado favorablemente para los partidos políticos que no habían detentado el poder, y que constituían el sistema de oposición partidaria.

Asimismo, la competitividad, que asumimos metodológicamente como un recuento del pasado mediato o inmediato de la capacidad de actuación de uno o varios partidos, cuyo reflejo se manifiesta en la cantidad de votos obtenidos a su favor, y consecuentemente el número de escaños o puestos obtenidos como resultado de la elección, se encontró atada, y por ello constreñida, en buena parte, a condiciones históricas desfavorables de la competencia, como consecuencia de un sistema político-electoral autoritario y excluyente, propio de un régimen de partido único primero, y de partido hegemónico después.

Por ello, la competitividad es un recurso plausible para evaluar la eficacia de los partidos políticos, así como también el grado en que se encuentra distribuida la fuerza política que representa al cuerpo electoral. Por este mismo curso, la competitividad permite asomamos a la capacidad de potencia que puede o pudo desplegar un partido político en un lapso determinado.

Por lo que hace a la medición de la competitividad de los partidos políticos en Baja California, se puede establecer lo siguiente:

a) El indicador "triunfos obtenidos", y el denominado "número de partidos", nos indican la traducción de la competitividad en la ocupación real de puestos de elección popular. 
b) El indicador "margen de victoria", y el denominado "potencia opositora", indican la confrontación de los datos y, por lo tanto, de la eficacia interpartidaria.

c) El indicador "expansión del sistema electoral" refleja un proceso evolutivo de un viaje paralèlo de los distintos partidos, cuya posibilidad de correlación es fundamentalmente con la evólución del padrón o del listado nominal.

Por todo ello, podemos inferir lo siguiente:

1. En términos generales, en la elección de 1997, el bipartidismo fue la nota distintiva en Baja California, donde el PAN y el PRI se enfrascaron en una lucha real por obtener el mayor número de votos, así como el mayor número de puestos de elección. Sin embargo, este bipartidismo es atenuado, ya que de seis puestos de elección (100\%), el PAN obtiene cinco $(83.3 \%)$, mientras que el PRI obtiene uno solamente, significando apenas $16.7 \%$.

Lo anterior permite afirmar que el proceso pasado de 1997 se caracterizó como uno no competitivo (la calidad competitiva sería aquélla en la cual participaron más de dos fuerzas con posibilidades reales de obtener un resultado electoral equilibrado, y cuyos puestos de elección hubiesen sido objeto de una distribución más o menos equitativa entre tres o cuatro actores políticos).

Sin embargo, es necesario señalar que no obstante la precariedad de la competitividad de los partidos políticos, ningún puesto de diputado de los distritos disputados fue obtenido por mayoría absoluta, es decir, con una votación mayor a $50 \%$.

2. Por lo que se refiere a los indicadores "triunfos obtenidos" $y$ "número de partidos", mencionados anteriormente, podemos observar, en forma por demás evidente, que la competitividad se reduce a dos partidos solamente, ya que en ambos casos, traduciéndose la mayoría de los votos obtenidos en escaños o puestos de elección, solamente el PAN logró $86.3 \%$ de ellos, y el PRI únicamente $16.7 \%$, sumando ambos $100 \%$ de los puestos de elección en disputa (no obstante que fueron ocho partidos políticos los que participaron en el proceso electoral). Bajo esta perspectiva, se puede decir que un partido político (el PAN) fue muy eficaz; un segundo partido (el PRI) fue ineficaz, y los seis partidos restantes (PRD, PT, PDM, PVEM, PPS y PC) fueron definitivamente muy ineficaces.

3. Por lo que se refiere a los indicadores "margen de victoria" y "potencia opositora", Ios cuales miden la eficacia de los partidos o del partido triunfador en relación con otro o al resto de los que participaron con él, se puede sintetizar diciendo que la competitividad de los partidos entre sí por la disputa de los votos es casi imexistente, salvo lo referente al 
PAN y al PRI, en ese orden. En lo que respecta al indicador "margen de victoria", en los cinco distritos que ganó el Partido Acción Nacional, su margen menor en relación con el PRi fue de $17.4 \%$, alcanzando incluso un margen de victoria en otro distinto de $54.7 \%$ con respecto al PRI. Ello nos habla de que en los cinco distritos que ganó Acción Nacional, en ninguno de ellos el PRI, ni el resto de los partidos, fueron competitivos en su lucha electoral contra el partido triunfador.

Por otra parte, la medición de la "potencia opositora" no hace sino reiterar lo ya señalado; es decir, que la suma de los partidos políticos perdedores, apenas equilibra a los votos obtenidos por el partido triunfador (me refiero especialmente a los distritos en los cuales triunfo el Partido Acción Nacional), lo cual nos habla de la baja competitividad de los partidos políticos de oposición para atraer votos a su favor, y cabe aclarar, incluso, que la mayoría de la potencia opositora que se suma a los partidos pequeños, corresponde al Partido Revolucionario Institucional.

4. El indicador "expansion del sistema electoral", muestra que durante el periodo 1994-1997, la dinámica del listado nominal, sobre todo en aquellos distritos donde se manifestó un crecimiento, incluso muy notorio como es el caso de los distritos 02,05 y 06 , los partidos políticos analizados (PAN y PRI) manifestaron dinámicas de crecimiento no correspondientes con el signo y/o la magnitud de la expresada por el listado nominal. De ellos, solo el PRD manifestó una mayor eficacia, al crecer incluso de manera más favorable e intensa que el propio listado nominal.

En este sentido, una de las cosas que sugiere este indicador, es que el sistema electoral en Baja California se dinamizó en forma no proporcional; es decir, que a un crecimiento de los electores no correspondió en forma directa un crecimiento electoral de los partidos políticos durante el proceso electoral de 1997. 


\section{BIBLIOGRAFÍA}

BECERRA, Ricardo, et.al. 1997, La reforma electoral de 1996, Ed. Fondo de Cultura Económica, México.

HERMET, Guy. 1997. ¿Para qué sirven las elecciones?, Ed. Fondo de Cultura Economica, México.

LAGROYE, Jacques. 1994. Sociología política, Ed. Fondo de Cultura Económica, Buenos Aires.

LIJPHART, Arend. 1995. Sistemas electorales y sistema de partidos, Ed. Centro de Estudios Constitucionales, Madrid.

MOLINAR Horcasitas, Juan. 1991. El tiempo de la legitimidad, Ed. Cal y Arena, México.

NOHLEN, Dieter. 1994. Sistemas electorales y partidos políticos, Ed. Fondo de Cultura Económica, México.

PANEBLANCO, Ángelo, 1990. Modelos de partido, Ed. Alianza, Madrid. ROUQUIE, Alain. 1986. ¿Para qué sirven las elecciones?, Ed. Fondo de Cultura Económica, México.

SARTORI, Giovanni. 1987. Partido y sistemas de partidos, libro I, Ed. Alianza, Madrid.

VALDEZ Zurita, Leonardo. 1995. Política y cultura, revista núm. 5, Ed. UAM-Xochimilco, México.

Código federal de instituciones y procedimientos electorales. 1997. Instituto Federal Electoral, México. 